\section{Trayectorias juveniles e intervenciones sociales: repercusiones en las prácticas sociales y en la salud (Río de Janeiro, Brasil)}

\author{
Juvenile trajectories and social interventions: \\ repercussions for social practices and health \\ (Rio de Janeiro, Brazil)
}

Simone Monteiro 1

Fátima Cecchetto 1

\footnotetext{
${ }^{1}$ Instituto Oswaldo Cruz, Fundação Oswaldo Cruz, Rio de Janeiro, Brasil.

Correspondencia S. Monteiro

Laboratório de Educação em Ambiente e Saúde, Departamento de Biologia, Instituto Oswaldo Cruz, Fundação Oswaldo Cruz. Av. Brasil 4365,

Rio de Janeiro, $R J$ 21040-900, Brasil. msimone@ioc.fiocruz.br
}

\begin{abstract}
This article analyzes the perceptions of a group of youth from the lower economic levels of Rio de Janeiro, Brazil, about the repercussions of social programs on their affective/sexual and educational/professional trajectories. From a comparative perspective, we examined the perspectives, practices, and conditions of life of 42 boys and girls, between 18 and 24 years of age, who did and did not participate in social projects directed at professionalizing and promoting the population. In particular, we discuss the impacts of interventions on concepts of health and AIDS, on indicators of social mobility and professionalization, and on the formation of social networks, including the dimension of gender. We sought to address the effects of civic organizations on the low-income youth population, in the context of socioeconomic crisis and of few social policy advances. We emphasize that in spite of the limitations of such interventions in the greater social context, the experience of social support promoted by the projects contributes to the expansion of life perspectives and the symbolic capital of this social group, with positive impacts for the health field.
\end{abstract}

Social Support; Social Programs; Youth

\section{Introducción}

En este artículo analizamos en qué medida la participación en programas sociales, dirigidos a la capacitación profesional y educación para la ciudadanía, influencia en las condiciones de vida, visiones y prácticas sociales y en salud de jóvenes de los estratos populares. Se trata de un estudio cualitativo sobre los efectos de intervenciones sociales en la trayectoria escolar/profesional y afectivo/sexual de un grupo de chicos y chicas de Río de Janeiro, Brasil.

Las investigaciones recientes sobre el comportamiento sexual y reproductivo de los jóvenes brasileños, implicando la franja de edad de 15 a 24 años, confirman los efectos de las desigualdades sociales y de género en las experiencias juveniles. Los datos atestiguan que los grupos de menor renta y escolaridad tienen una iniciación sexual más temprana, vivencian la maternidad/paternidad más precozmente, usan preservativo con menos frecuencia y revelan mayor desconocimiento sobre el SIDA, cuando se comparan con jóvenes de mejor renta y escolaridad 1,2 . Nos indican dos tendencias que las prácticas sexuales desprotegidas han sido más frecuentes entre la población juvenil empobrecida. Una de ellas es la mayor ocurrencia de casos de embarazo en estos estratos sociales, a pesar de la caída en las tasas de fecundidad de Brasil en los últimos cuarenta años 3 . La otra se refiere a la importancia de la vía sexual 
como categoría de exposición al VIH $(67,3 \%$ del total de casos en 2004 entre hombres y $94,9 \%$ entre mujeres) conjugada con los indicadores nacionales de la mayor vulnerabilidad al virus del SIDA entre los grupos de bajo poder adquisitivo ${ }^{4}$. En términos generacionales la tasa de incidencia es más expresiva en la franja de 25 a 34 años. No obstante, se puede inferir que una parte significativa de la infección se produce en el proceso de transición a la vida adulta, debido al largo período de incubación del virus (cerca de diez años).

La variación significativa en la razón de casos entre hombres y mujeres de 24,2 en 1985 , a 1,5 en 2004 ilustra las diferencias de género y revela el aumento de casos de VIH/SIDA entre mujeres. En la franja de 13 a 19 años este fenómeno es particularmente evidente, pues desde 1998 la cantidad de chicas notificadas ha sido mayor que la de los chicos 4 . Contribuye a la comprensión de este escenario, el caso de la iniciación sexual femenina con hombres más viejos, la no utilización del preservativo en las llamadas relaciones estables y el valor del embarazo como un bien simbólico, conforme muestran los estudios en el área de las ciencias sociales sobre sexualidad, reproducción, género y juventud 5,6,7. Tales investigaciones han llamado la atención sobre la importancia del examen de la dinámica de factores socioeconómicos, históricos y culturales involucrados en la definición de las prácticas de lo cotidiano y, en consecuencia, aquellas relacionadas con la salud sexual y reproductiva de los jóvenes.

Demás reflexiones sobre la tematización de la juventud en organizaciones sociales y en la producción académica confirman la necesidad de avanzar en los planteamientos centrados en la contextualización histórico social de las experiencias juveniles, relativas a la sexualidad y reproducción. Todavía predomina un punto de vista universalizante y naturalizador en el que esa fase de la vida tiende a ser analizada primordialmente como un fenómeno estructuralmente psicobiológico ${ }^{8}$. Del mismo modo, las diferencias de comportamiento, derivadas de las desigualdades sociales y de género presentes en esta categoría social-edad, en general, no son contempladas en los planteamientos preventivo/ educativos sobre prevención del VIH/SIDA. Estas son caracterizadas por el énfasis en la transmisión de la información, en la responsabilización individual, en el uso del preservativo y en la producción de materiales educativos 5,9. De esta forma, se distancian de las singularidades simbólicas de los grupos sociales y no actúan en la disminución de la vulnerabilidad social y económica, que alimentan las iniquidades sociales.
La diversidad y las formas de construcción de la categoría juventud, igualmente son un desafío para las políticas sociales destinadas a los jóvenes de las capas populares. Entre educadores y agentes sociales predomina el encuadramiento de los comportamientos juveniles como a priori "desviantes" o de "riesgo", sobre todo entre las poblaciones empobrecidas. En este particular, resaltamos el peligro de criminalizarse o estigmatizar las prácticas juveniles de este segmento, dejando de identificar actitudes y comportamientos fundamentados en otras premisas 10,11 .

Desde otra perspectiva, vienen emergiendo, principalmente en el ámbito de la sociedad civil organizada, iniciativas orientadas por la concepción de una construcción participativa, conjugando actividades que estimulan la creatividad y la capacidad del joven de tener una conciencia crítica sobre el mundo 12,13. Lo importante es saber cuáles son sus efectos en las condiciones materiales de existencia, en las percepciones y prácticas sociales y en salud de los jóvenes involucrados. En esta dirección, este artículo tiene el propósito de analizar la repercusión de cuatro proyectos sociales, realizados por instituciones gubernamentales y de la sociedad civil de Río de Janeiro, en la trayectoria biográfica de chicos y chicas.

\section{Metodología}

El estudio incluyó la realización de entrevistas profundizadas a 21 chicas y 21 chicos en la franja de edad de 19 a 24 años. De los 42 entrevistados, 24 habían participado en proyectos sociales y 18 no habían tenido esta experiencia, pero ambos presentaban características semejantes en relación al sexo, edad, inserción social y lugar de vivienda. La comparación de los datos de una población con características de edad y socioeconómicas semejantes pretende indicar tendencias relacionadas con la influencia de experiencias sociales en las trayectorias de vida del grupo en cuestión. Fueron también realizadas entrevistas con los educadores de los proyectos y una observación directa de las actividades educativas.

Los proyectos seleccionados tienen en común los objetivos generales, dirigidos a la promoción de la ciudadanía y la profesionalización de grupos de bajo poder adquisitivo, con prioridad para la franja de 14 a 21 años, siendo que algunos enfatizan la cualificación en el ámbito artístico y cultural y otros la formación técnica.

La mirada conferida al tema privilegió los sistemas de representaciones y prácticas socia- 
les del universo seleccionado, dentro de la tradición socio-antropológica, caracterizada por el estudio y descripción detallada de valores y prácticas de grupos sociales demarcados 14,15.

\section{Discusión}

En el análisis de las entrevistas buscamos identificar en qué medida las visiones, las prácticas y las condiciones de vida de los/as 24 jóvenes con participación en proyectos sociales se diferencia de aquellos/as 18 sin experiencia. Elegimos las concepciones de salud y SIDA, los indicadores de movilidad social, profesionalización y la formación de redes sociales, incluyendo la cuestión de género. La dimensión étnico/racial no será tratada en este artículo. Los jóvenes con participación en proyectos sociales serán de ahora en adelante denominados "P" y los sin experiencia "NP".

\section{Perfil del grupo}

Los/as jóvenes entrevistados/as son habitantes de favelas y/o barrios populares de la Ciudad de Río de Janeiro. Gran parte vive en estos lugares desde su nacimiento, indicando la permanencia del grupo en sus familias de origen, la mayoría de la Región Sudeste. La mitad se declaró soltera y sólo diez jóvenes afirman mantener una unión estable. En la auto-clasificación de color/raza, la mitad respondió negro, un tercio se clasificó como moreno y una minoría como blanca. La menor proporción de blancos en este grupo es coherente con las estadísticas nacionales sobre la mayor concentración de negros y morenos en los estratos poblacionales de menor poder adquisitivo. Todos ya frecuentaron la escuela, pero un poco más de la mitad continúa estudiando. Aunque no haya diferencia significativa entre los jóvenes $\mathrm{P}$ y NP, aquellos/as que participaron en los proyectos presentan mejor nivel de escolarización. Para tres, de los cuatro proyectos, la escolaridad no es la condición para la inserción en el proyecto, pero hay un fuerte estímulo a la enseñanza formal por medio de actividades de refuerzo escolar.

El valor de la escolarización fue asociado a la adquisición de conocimiento y a la ampliación de las perspectivas profesionales. Entre aquellos que están estudiando, las diferencias de género aparecieron por la presencia de hombres en los cursos profesionalizantes, en oposición a ninguna mujer. Los chicos y chicas que salieron de la escuela, a semejanza con otros hallazgos, alegan en común la falta de estímulo, siendo que entre los hombres hay más refe- rencias al problema financiero, mientras entre las mujeres aparece el embarazo.

\section{Salud y SIDA}

En lo que se refiere a las visiones e informaciones sobre la epidemia de VIH/SIDA observamos el mayor dominio de los/de las jóvenes $\mathrm{P}$ en relación a los NP. Aquellos/as que integraron los proyectos tienden a reconocer que la vulnerabilidad al virus deriva de la no adopción de prácticas preventivas y que no está restringida a grupos sociales específicos. Además refieren con más frecuencia que el uso del preservativo posibilita la práctica sexual sin riesgo. Los/as jóvenes NP, a su vez, dan más énfasis a la pareja fija y a las relaciones de confianza como forma de protección al VIH 5. Las mujeres NP son las que más afirman no usar cualquier medio de prevención, además de ser las que tienen menos información sobre el SIDA y otras enfermedades sexualmente transmisibles.

Cuando se les pregunta acerca de las fuentes de información sobre ETS y SIDA, los/as jóvenes NP hacen referencia a los medios de comunicación, a la escuela y los amigos/familia. Ya jóvenes $\mathrm{P}$ citan con mayor frecuencia el propio proyecto, seguido de la escuela y de los servicios de salud. Las orientaciones en salud están asociadas a ponencias y conversaciones continuas con los educadores (ellos hablan siempre), indicando el énfasis en el carácter informativo y en el diálogo, que podrá ser profundizado a partir del análisis de las prácticas educativas de los proyectos, que se realizará posteriormente.

En el universo de los/as jóvenes del proyecto, los cambios en el área de la salud fueron atribuidos a la adquisición de nuevos hábitos relativos a los cuidados con el cuerpo ("hacer ejercicios"; "antes yo no me preocupaba de tomar un baño muchas veces al día") y la alimentación ("antes no prestaba mucha atención a eso de comer, era muy dejada. Comía en cualquier lugar"). Identificamos incluso en los relatos la noción de prevención de enfermedades, relacionada con la búsqueda de atención médica y al uso del preservativo, principalmente entre las mujeres, denotando la presencia de una dimensión más moderna, centrada en la idea del cuidado de sí mismo.

Es necesario verificar en qué medida el conocimiento mencionado por los jóvenes se traduce en prácticas preventivas. Aunque esta discusión rehuya del objetivo de este trabajo, los datos sobre reproducción pueden ofrecer algunas pistas. 


\section{Reproducción}

De los 38 jóvenes con experiencia sexual, la mayor parte vivenció el embarazo (15 mujeres y 9 compañeras de los chicos entrevistados) y cerca de la mitad tuvo hijos. La mayoría es mujer, situación convergente con los hallazgos de las estadísticas nacionales sobre la tendencia de las mujeres a ser madres en edad más precoz que los chicos 2 . A excepción de tres chicas que entraron en el proyecto por ser madres jóvenes en situación de precariedad, los/as jóvenes $\mathrm{P}$ tuvieron menos hijos que los NP, aunque la diferencia no sea expresiva. En general, la crianza de los niños es asumida por las madres del bebé. Sin embargo, entre los/as jóvenes $\mathrm{P}$ hay menciones a la participación del padre, sugiriendo la presencia de una perspectiva más moderna de las relaciones de género. En consonancia con demás estudios 5,6,7 predominan percepciones positivas acerca de la materni$\mathrm{dad} /$ paternidad, expresada por la recurrencia de la respuesta "mi hijo representa todo". Los cambios más comunes son aumento o adquisición de responsabilidad.

En la definición del momento ideal para quedarse embarazada prevalece el énfasis en las condiciones materiales (ej. empleo, estabilidad financiera, casa propia), traducida en comentarios bastante idealizados sobre la necesidad de estructurarse o tener estabilidad financiera antes de tener hijos. Tal dato puede indicar la internalización de la noción moderna de maternidad/paternidad responsable. No obstante, el modelo tradicional de género se presenta en la aproximación entre el impulso sexual y la masculinidad en la visión de los chicos y en la asociación entre emoción y compromiso en la perspectiva femenina, dado que apenas los hombres atribuyen el momento ideal del embarazo al deseo, mientras las mujeres resaltan el vínculo emocional y el matrimonio.

\section{Significado del trabajo y profesionalización}

Cerca de la mitad (23) de los/as jóvenes investigados declaró estar trabajando. De estos, la mayor parte (10 hombres y 7 mujeres) había participado en los proyectos sociales y estaba ejerciendo actividades vinculadas a ellos (monitores, locución, percusión, actor) u ocupados en el sector de servicios (farmacias, supermercados, confecciones). Comparativamente, los jóvenes sin experiencia en proyectos estaban más representados en el sector informal y precario del mercado de trabajo (vendedor ambulante, albañil, recoge basura, trabajador en tareas de poca monta) o desempleados. El desem- pleo es más abundante entre las mujeres NP, condición semejante a los estudios respecto a los significativos cambios en el mundo del trabajo y sus resultados de selectividad y exclusión que alcanzan particularmente a las mujeres de los segmentos pobres 16. Estos datos confluyen con la hipótesis de que los proyectos sociales contribuyen a la ampliación de las oportunidades de inserción profesional.

En cuanto a los beneficios proporcionados por el trabajo, los jóvenes $\mathrm{P}$ indicaron principalmente la ampliación de las perspectivas personales, a partir de la cualificación profesional (conseguí experiencia) y de los contactos (las puertas se abrieron) acreditados a la participación en los proyectos. Además de la renta, las actividades laborales representaron la oportunidad de adquisición de conocimiento y crecimiento personal, como se delinea en el habla de una joven: "hago aún más cosas en el trabajo. No es sólo quedarse todo el tiempo en la computadora, hay que entregar un informe. Voy aprendiendo con los otros y con el trabajo. A veces surge una pregunta de una visitante, que no sabía algo, entonces pregunta, (...). Voy aprendiendo más y más".

Vale resaltar que para el grupo de jóvenes $\mathrm{P}$ el "estar trabajando" también resulta en una garantía de "respeto" y "honra" en la convivencia social, configurando un significado ético al trabajo. La centralidad de la actividad remunerada apareció de forma diferenciada entre los jóvenes NP. En este grupo, el trabajo se caracterizó más como una necesidad de "ocupar el tiempo" dada la situación de mayor precariedad y desprotección social. Recordamos que para algunos jóvenes ingresar en el circuito de los proyectos sociales emergió como alternativa o "refugio" a la violencia, conforme revela la declaración de un chico que integró un proyecto.

"Yo esnifaba disolvente en esa época [de la calle]. Y fui viendo que fui cambiando [ya había sido preso]. Paré de esnifar disolvente, volví a casa de mi abuela, mi vida fue cambiando de una manera frenética... Entonces conseguimos montar nuestra propia radio aquí, con unos equipamientos (...). A partir de ahí comenzamos a ser conocidos; a hacer entrevistas. Luego mandamos este proyecto, la alcaldía lo aprobó, nos dio una subvención, compramos equipamientos nuevos...".

En el universo investigado, constatamos la valorización de la distinción social promovida por el compromiso con el circuito artístico-cultural, ya indicado en otros análisis sobre la contribución de los proyectos sociales en la difusión de nuevas identidades juveniles, basadas en estilos globalizados 17. En este particu- 
lar, los chicos forman el grupo que más asoció la participación en los proyectos al aumento del status en la comunidad y en su círculo social más amplio. Para ellos, el trabajo es tenido como de suma importancia no sólo como proveedor de oportunidades, sino como espacio de construcción de identidad 18. Hay una búsqueda de actividades capaces de producir una gratificación por su desempeño y, también, elevar su posición en la jerarquía de prestigio en su contexto relacional.

\section{Movilidad y redes sociales:} permanencias y cambios

En términos de movilidad social, las declaraciones de los/as jóvenes $\mathrm{P}$ y NP sobre el conocimiento de personas del círculo familiar o de amigos que cambiaron de vida revelan que el grupo - como un todo - reconoce una significativa ascensión a los que integraron los proyectos.

Al ser instados a comparar las propias trayectorias de vida con la de sus padres, la mayor parte de los $\mathrm{P}$ que respondieron afirmó que sus padres vivían en peores condiciones que ellos cuando tenían la misma edad. Las principales evidencias se refieren a la menor cantidad de hijos, a la adquisición de bienes materiales, a las mejores condiciones de vivienda y al distanciamiento del lugar de origen. Según la visión de los NP, sin embargo, sus padres llevaban una vida en una situación más favorable, destacando la ausencia de desempleo "tenían más oportunidades" y de la violencia urbana “en aquella época no había tráfico de drogas".

La percepción de los jóvenes $\mathrm{P}$ sobre su ascenso social se aproxima a los estudios sociológicos que consideran la movilidad intergeneracional como un indicador de cambios en la posición en el sistema de estratificación social 16. No obstante, según la literatura, estos movimientos de corta distancia entre las generaciones, no promueven el impacto en las desigualdades estructurales. Tal hecho se debe, en parte, al estilo de desarrollo económico del país y la fragilidad de las instituciones que representan canales tradicionales de movilidad como la escuela. Se resalta incluso que, a pesar del incremento en la escolaridad de las nuevas generaciones, permanece el problema de la inserción en el mercado de trabajo para los jóvenes con cualificación insuficiente en las sociedades contemporáneas, agravado por las alteraciones en las expectativas juveniles 19.

Para varios chicos y chicas de las capas populares la participación en la red social abierta por las actividades de los proyectos significó la posibilidad de romper con la red cercana, mo- dificando y expandiendo el círculo de relaciones. Por otro lado, esa ampliación también se refiere a la entrada en "territorios" considerados enemigos, o sea, en comunidades sometidas a la dominación violenta de los traficantes en que la simple circulación de personas de otras áreas representa peligro de vida 20 .

En la definición de sus principales problemas, los/las jóvenes NP, principalmente las mujeres, indicaron la falta de dinero y las cuestiones familiares. En este mismo grupo, la familia, notablemente la madre, continúa siendo la referencia principal como soporte emocional y financiero. Entre los/as jóvenes P el trabajo es más referido como una fuente de preocupación, mientras que la red de amigos y los “contactos" favorecidos por los proyectos son apuntados como una especie de retaguardia frente a situaciones problemáticas. Los datos sugieren que mientras los/as jóvenes NP conservan los vínculos con el núcleo familiar, en función de opciones económicas restrictas, aquellos/as con experiencia en los proyectos pueden echar mano de un campo de posibilidades, estableciendo nuevas relaciones con el mundo a su alrededor. En esta cuestión, no se puede ignorar la influencia de una concepción tradicional en la demarcación rígida de papeles de género, determinando que el hombre asuma desde pronto su función de proveedor, además de estímulo a la independencia de los vínculos familiares y afectivos.

Según los/as jóvenes $\mathrm{P}$ sucedieron transformaciones importantes en la auto-imagen, descrita también como "apariencia”. Una chica afirmó "cambió mi forma de vestir, andaba (...) toda sucia, con el pelo de punta. Hoy en día procuro arreglarme”. Un chico dijo “(...) antes era dejado con la apariencia; andaba descalzo todo sucio, hay que estar a gusto pero la buena apariencia es importante". Las declaraciones revelan que los/as jóvenes están en sintonía con la creciente importancia que la moda y el vestuario tienen en su construcción de identidad.

Otra transformación percibida entre los integrantes de los proyectos, fue con la actitud de la familia. Algunos relatos dan cuenta de que los familiares "percibieron la diferencia" y pasaron a "tener orgullo por el esfuerzo", confiriendo legitimidad a las acciones de los proyectos. Complementa este punto de vista, la percepción de algunos jóvenes acerca de los cambios en la manera de relacionarse en casa. Hay declaraciones sobre el aumento del diálogo entre los padres (procurar conversar más; antes yo no conversaba tanto con mi padre) y de la convivencia con personas de la familia (se quedaba más en casa). 
Sin embargo, algunos identificaron el crecimiento de la animosidad y de la desconfianza de la red de vecindad cercana. Según afirmaron, la visibilidad derivada de la inserción en el proyecto, provocó reacciones ambiguas de los pares con quejas para que se retornase a padrones de sociabilidad anteriores a la entrada en el proyecto. Es decir, las visiones sobre las alteraciones también fueron percibidas por familiares y vecinos de forma negativa, como si el vínculo con el proyecto remitiese a los jóvenes a otro mundo, distante de la realidad y de los valores locales. Contribuyó incluso a una diferenciación en relación con la realidad de las localidades de origen, el incentivo al hábito de lectura, el manejo del ordenador y el acceso a Internet. Dentro de la noción de ampliación de horizontes, fue mencionado el conocimiento de otras realidades derivadas de visitas a ciudades, dentro y fuera del país.

\section{Conclusiones}

En base a las declaraciones, constatamos que, en la visión de los/as jóvenes, las intervenciones sociales promueven cambios en sus trayectorias de vida, traducidas por la elevación del padrón de vida y de las perspectivas de inserción profesional, por el estímulo a la escolarización y ampliación de las redes de sociabilidad. Podemos inferir que, en un contexto de carencia de bienes materiales, simbólicos y de falta de protección social, la experiencia del apoyo social (personal y profesional) promovida por los proyectos asume un valor positivo, a pesar de las limitaciones de tales intervenciones en el contexto macro social.

Además, suponemos que la identificación de las oportunidades ocupacionales e intelectuales abiertas por medio de la intervención, en alguna medida, se coloca como una alternativa frente a las situaciones de abandono social, a la violencia social y a la entrada de los jóvenes en el circuito de la criminalidad y del tráfico. Este punto de vista encuentra convergencias con la reflexión de Novaes 21 acerca de los efectos de organizaciones civiles para la población juvenil de baja renta. Según palabras de la autora 21 (p. 153): “(...) estos jóvenes aportan un capital simbólico para toda la comunidad (...) $y$, en consecuencia, son cuestionados los límites que interiorizan (y hacen naturales) los mecanismos de exclusión y segmentación social. Parte de ellos se convierten en 'profesionales de los Proyectos', adquieren una competencia específica para otro tipo de mediación entre los jóvenes de las áreas pobres y las instituciones externas".
Otra indicación del análisis se refiere a las evidencias sobre el mayor conocimiento de los integrantes de los proyectos en el área de la salud sexual y reproductiva, cuando se compara con los relatos de los jóvenes NP, en lo que se refiere a: las ETS/SIDA, la percepción de riesgo individual, y la valorización del uso del preservativo, entre otros. Tales datos ganan significado cuando se observa un mayor cuidado de este grupo con la salud y el control de la reproducción, expresada por el adelantamiento de la maternidad/paternidad y por la mayor preocupación con la crianza de los hijos. Comprendemos que las contribuciones relativas al acceso de la información, a los medios de prevención y a las orientaciones sobre el tema podrán ser mejor comprendidos a partir del análisis de las actividades de las organizaciones y de la profundización en las trayectorias de vida de los entrevistados.

Los indicadores de movilidad social de los/ as jóvenes se expresaron por las posibilidades de movimiento en el mercado de trabajo y por la relativa ascensión social intra-generacional e intraclase. La identificación de beneficios no significa ignorar los límites de esas acciones, sea en relación a su alcance junto a la población joven urbana de bajo poder adquisitivo, sea en términos de su impacto para la disminución de las desigualdades sociales. Es decir, reconocemos que los proyectos investigados están restringidos a un grupo minoritario y no producen un impacto en las estructuras fomentadoras de las condiciones desiguales de existencia. Sin embargo, en el análisis de los límites de las intervenciones es preciso tener en vista que el bajo crecimiento económico y las exigencias cada vez mayores de ingreso en una sociedad de consumo vienen produciendo, en la historia brasileña, un significativo proceso de movilidad descendente para las nuevas generaciones. Son en los sectores más pobres de la población donde se presentan las mayores dificultades de estabilización social. Los tradicionales canales de movilidad social como la educación y el trabajo, cada vez más cerrados, han sido substituidos por la integración de los jóvenes en redes sociales que confieren importancia creciente a las actividades de ocio y consumo en la definición de nuevas identidades.

En este sentido, no es posible ignorar la vitalidad de las actividades que ocurren fuera del modelo dominante, tanto del sistema económico formal como del Estado. La invención cotidiana de identidades y sociabilidades por los llamados sectores excluidos ha sido frecuentemente viabilizada por las organizaciones no gubernamentales, esfera que tiene la capaci- 
dad de representar o expresar necesidades de grupos locales ante el público nacional e internacional y de movilizar valores solidarios, además de ser una fuente de empleo y espacio de participación para estos grupos 22 . Esto significa decir que compartimos la presuposición de que determinados proyectos sociales dedicados a la población juvenil pueden contribuir a la ampliación de la perspectiva de vida y del capital simbólico de este grupo social, con impactos positivos para la esfera de la salud, ante un contexto de crisis socioeconómica y de los pocos avances en términos de políticas sociales y de seguridad pública.

El desafío para los jóvenes brasileños es cómo constituirse en sujetos con perspectivas de cambios que limiten la exclusión, ante la situación de precariedad social experimentada en las últimas décadas. El análisis aquí desarrollado visa estimular nuevas reflexiones sobre el papel actual de las intervenciones sociales en el enfrentamiento de las desigualdades socioeconómicas que hacen la vida de segmentos poblacionales un drama cotidiano.

\section{Resumen}

El artículo analiza la percepción de un grupo de jóvenes de las capas populares de Río de Janeiro, Brasil, sobre las repercusiones de programas sociales en sus trayectorias afectivo/sexual y escolar/profesional. Desde una perspectiva comparativa, examinamos las visiones, las prácticas y las condiciones de vida de 42 chicos y chicas, entre 18 y 24 años, con y sin participación en proyectos sociales dirigidos a la profesionalización y promoción de la ciudadanía. Son discutidos, particularmente, los impactos de las intervenciones en las concepciones de salud y SIDA, en los indicadores de movilidad social y profesionalización y en la formación de redes sociales, incluyendo la dimensión de género. Buscamos reflexionar sobre los efectos de organizaciones civiles en la población juvenil de baja renta, en un contexto de crisis socioeconómica y de pocos avances en términos de políticas sociales. Destacamos que, a pesar de las limitaciones de tales intervenciones en el contexto macro social, la experiencia de apoyo social promovida por los proyectos contribuye a la ampliación de la perspectiva de vida y del capital simbólico de este grupo social, con impactos positivos para la esfera de la salud.

Apoyo Social; Programas Sociales; Juventud

\section{Colaboradores}

El trabajo de campo, la redacción y la revisión final fueron realizados por ambas autoras.

\section{Agradecimientos}

Nos gustaría agradecer la colaboración de las investigadoras Cristina Castello Branco, Danielle Moraes y Sandra Rebello en la realización de las entrevistas y análisis de los datos, así como al grupo de jóvenes entrevistados y los profesionales de los proyectos sociales estudiados. 


\section{Referencias}

1. Calazans G. Os jovens falam sobre sua sexualidade e saúde reprodutiva: elementos para reflexão. In: Abramo HW, Branco PM, organizadores. Retratos da juventude brasileira. Análise de uma pesquisa nacional. São Paulo: Editora Fundação Perseu Abramo/Instituto Cidadania; 2005. p. 215-41.

2. Aquino EML, Heilborn ML, Knauth D, Bozon M, Almeida MC, Araujo J, et al. Adolescência e reprodução no Brasil: a heterogeneidade dos perfis sociais. Cad Saúde Pública 2003; 19 Suppl 2:S377-88.

3. Berquó E, coordenadora. Comportamento sexual da população brasileira e percepções do HIV/ AIDS. Brasília: Programa Nacional de DST/AIDS, Ministério da Saúde; 2000. (Série Avaliação 4).

4. Ministério da Saúde. Boletim Epidemiológico AIDS e DST 2004; Ano I, n. 1.

5. Monteiro S. Qual prevenção? AIDS, gênero e sexualidade em uma favela carioca. Rio de Janeiro: Editora Fiocruz; 2002.

6. Cabral C. Vicissitudes da gravidez na adolescência entre jovens das camadas populares do Rio de Janeiro [Dissertação de Mestrado]. Rio de Janeiro: Instituto de Medicina Social, Universidade do Estado do Rio de Janeiro; 2002.

7. Brandão E. Individualização e vínculo familiar em camadas médias. Um olhar através da gravidez na adolescência [Tese de Doutorado]. Rio de Janeiro: Instituto de Medicina Social, Universidade do Estado do Rio de Janeiro; 2003.

8. Calazans G. Cultura, adolescência e saúde: Argentina, Brasil, México. In: Oliveira MC, organizadora. Cultura, adolescência e saúde: Argentina, Brasil, México. Campinas: Consórcio de Programas em Saúde Reprodutiva e Sexualidade na América Latina; 1999. p. 44-97.

9. Monteiro S, Vargas E. Desafios teóricos no campo da prevenção. Divulg Saúde Debate 2003; 29:2433.

10. Abramo H. Considerações sobre a tematização social da juventude no Brasil. Revista Brasileira de Educação 1997; 5/6:25-36.

11. Moraes A. Os jovens e seus educadores: visões da sexualidade. In: Novaes R, Porto M, Henriques R, organizadores. Juventude, cidadania e cultura. Rio de Janeiro: Instituto de Estudos da Religião; 2002. p. 151-63.
12. Novaes R. Introdução. In: Novaes R, Porto M, Henriques $\mathrm{R}$, organizadores. Juventude, cidadania e cultura. Rio de Janeiro: Instituto de Estudos da Religião; 2002. p. 11-21.

13. Castro M, Abramovay M, Rua MG, Andrade E. Cultivando vida, desarmando violências: experiências em educação, cultura, lazer, esporte e cidadania com jovens em situação de pobreza. Brasília: Organização das Nações Unidas para a Educação, a Ciência e a Cultura/Brasil Telecom/Fundação Kellog/Banco Interamericano de Desenvolvimento; 2001.

14. Damatta R. Relativizando: uma introdução à antropologia social. Rio de Janeiro: Editora Rocco; 1987.

15. Becker HS. Métodos de pesquisa em ciências sociais. São Paulo: Editora Hucitec; 1994.

16. Scalon MC. Mobilidade social no Brasil: padrões e tendências. Rio de Janeiro: Editora Revan/Instituto Universitário de Pesquisas do Rio de Janeiro; 1999.

17. Zaluar A. Integração perversa: pobreza e tráfico de drogas. Rio de Janeiro: Editora Fundação Getúlio Vargas; 2004.

18. Guimarães N. Trabalho: uma categoria-chave no imaginário juvenil? In: Abramo $\mathrm{H}$, Branco $\mathrm{P}$, organizadores. Retratos da juventude brasileira. Análise de uma pesquisa nacional. São Paulo: Editora Fundação Perseu Abramo/Instituto Cidadania; 2005. p. 149-74.

19. Sansone L. Negritude sem etnicidade: o local e o global nas relações raciais e na produção da cultura negra. Salvador/Rio de Janeiro: EDUFBA; 2004.

20. Cecchetto F. Violência e estilos de masculinidade. Rio de Janeiro: Editora Fundação Getúlio Vargas; 2004.

21. Novaes R. Juventudes cariocas: mediações, conflitos e encontros culturais. In: Vianna H, organizador. Galeras cariocas: territórios de conflitos e encontros culturais. Rio de Janeiro: Editora UFRJ; 1997. p. 119-60.

22. Sorj B. A nova sociedade brasileira. Rio de Janeiro: Jorge Zahar Editor; 2000.

Recibido el 21/Jul/2005

Aprobado el 08/Ago/2005 